\title{
Effectiveness of Powell's Cognitive Rehabilitation with Neuro- feedback in Executive Functions, Memory, and Attention to Veterans with Post-Traumatic Stress Disorder
}

\section{ART ICLE INF O}

\section{Article Type}

Original Research

\section{Authors}

Sahragard M* PhD,

Alipour A. ${ }^{1} P h D$,

Zare $\mathrm{H}^{1} \mathrm{PhD}$,

Roshan R. ${ }^{2} P h D$,

Moudi M. ${ }^{3} M S C$

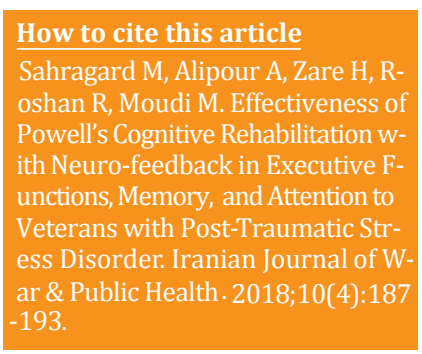

\section{A B S T R A C T}

Aims PTSD is a set of continuous and frequent symptoms that occur after experiencing or observing a traumatic event, such as being involved in a war. The aim of this study was to evaluate the effectiveness of Powell's cognitive rehabilitation with neurofeedback in improving executive functions, memory, and attention to veterans with post-traumatic stress disorder. Materials \& Methods This semi-experimental study, with pre-test post-test design, was carried out on 24 veterans with post-traumatic stress disorder in two experimental and control groups. Powell's cognitive rehabilitation treatment with neurofeedback was performed for 12 sessions and three months for the experimental group. For conducting the study, PSSI test, Wisconsin Card Test, Stroop test, and working memory test were used. Data were analyzed using univariate covariance analysis and SPSS 24.

Findings By controlling the pre-test effects, there was a significant difference between the experimental and control groups in terms of overall score of executive functions, memory and attention $(\mathrm{p}=0.001)$.

Conclusion Powell's cognitive rehabilitation treatment with neurofeedback is effective on improving executive functions, memory and attention of veterans with post-traumatic stress disorder.

Keywords Stress Disorders, Post-Traumatic; Rehabilitation; Neurofeedback; Executive Functions;
Memory; Attention

\section{I T A T I O N L I N KS}

[1] Mental disorders... [2] The prevalence of PTSD in conscript and... [3] Study of sleep quality in chemical-warfare-agents exposed ... [4] Executive function and PTSD ... [5] Gray matter density in limbic and paralimbic... [6] Neural correlates of emotion regulation ... [7] Caudate volumes in public ... [8] The impact of eye movement and tones on disturbing memories involving ... [9] Evaluation and comparison of executive functions ... [10] The association between complex trauma and ... [11] The role of executive function in posttraumatic ... [12] Executive function in posttraumatic stress disorder ... [13] Genome-wide analysis of mRNA targets for ... [14] Treating PTSD in patients with psychosis ... [15] Treating trauma in psychosis with EMDR: A pilot ... [16] The impact of eye movement and ... [17] How eye movementsin EMDRwork: Changes ... [18] Cognitive-Behavioral Therapy and Neuroscience ... [19] Functional Neurosurgery and Neuro-cognitive ... [20] Emotional, behavioral and cognitive mechanisms ... [21] Cognitive rehabilitation programs in schizophernia ... [22] Neurocognitive functioning in schizophernia ... [23] Neurorehabilitation of executive ... [24] EEvidence-based cognitive rehabilitation ... [25] Noninvasive cortical stimulation enhances ... [26] Plastic modulation of PTSD resting-state ... [27] Acquired self-control of insula cortex modulates emotion ... [28] Neurotherapy of traumatic brain ... [29] Feasibility and effectiveness of a cognitive ... [30] Effectiveness of computerized cognitive rehabilitation ... [31] Methods in behavioral ... [32] Evaluation of cognitive functions in patients ... [33] Study of information processing and some ... [34] Active memory function in female students with high ... [35] Transsynaptic modulation of kainate receptor ... [36] The efficacy of cognitive rehabilitation therapy: A meta-analytic ... [37] The effectiveness of computer-assisted cognitive ... [38] Social and nonsocial cognition in bipolar disorder and schizophrenia ... [39] Prefrontal cortex and executive function impairments in ... [40] Testing the effectiveness of group-based memory rehabilitation ... [41] The effectiveness of memory rehabilitation following ... [42] Improving memory in outpatients with neurological disorders using a ... [43] Cognitive training for memory deficits in stroke ...

\section{Article History}

Received: February 07, 2018

Accepted: July 17, 2018

ePublished: October 11, 2018 
تعريف DSM-5 رويداد آسيبزا شامل مرك، جراحت جدى، تجاوز

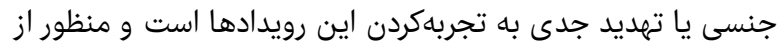

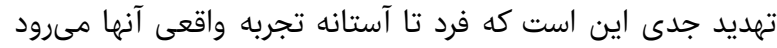

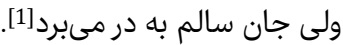

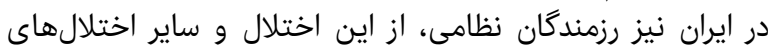

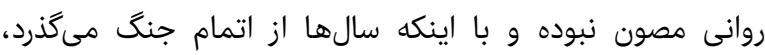

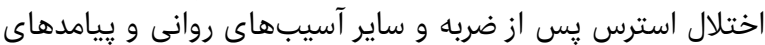

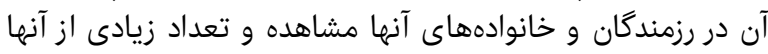

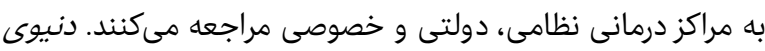

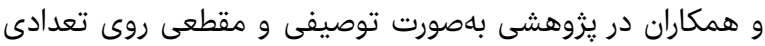

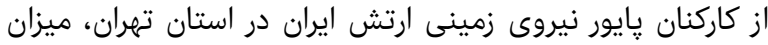

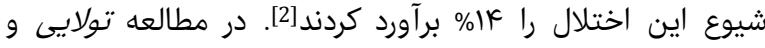

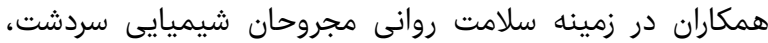

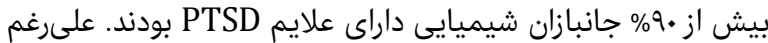

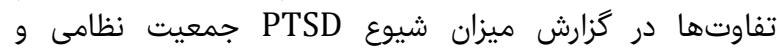

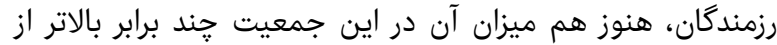

جمعيت عادى است [3].

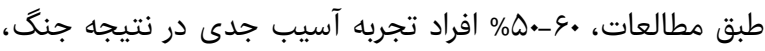

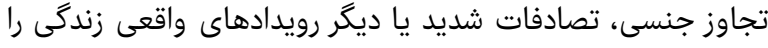

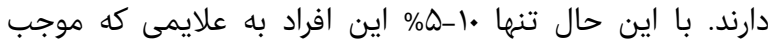

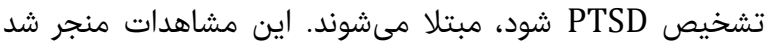

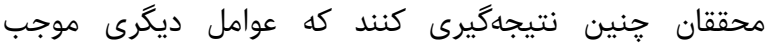

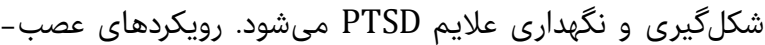

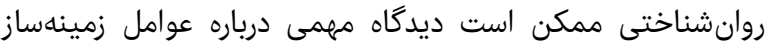

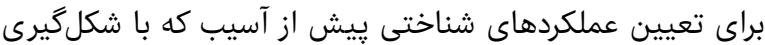

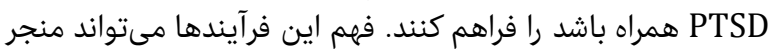

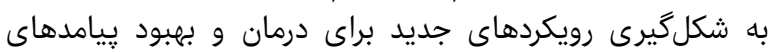

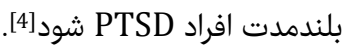

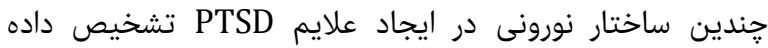

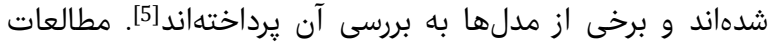

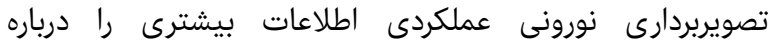

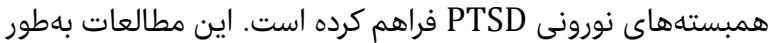

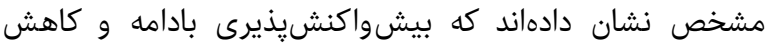

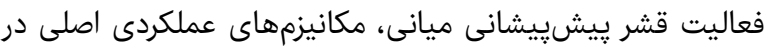

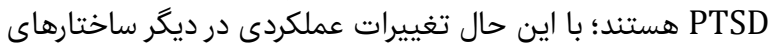

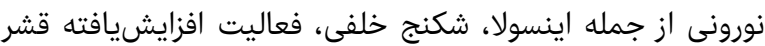

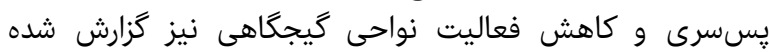

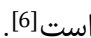

تغييرات ساختارى مانند كاهش ماده خاكسترى ممكن است

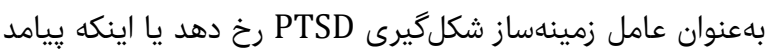

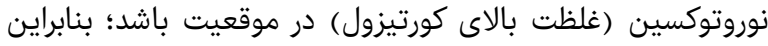

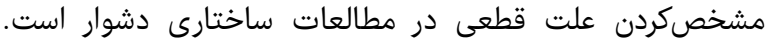

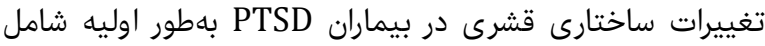

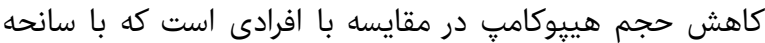

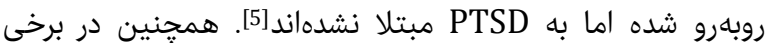

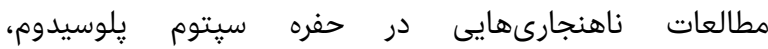

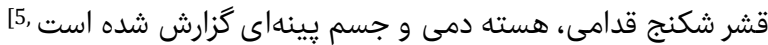

طبق مطالعات عصب- روانشناختى، بهدنبال مشكلات ساختارى، PTSD با نقايص ههمى در عملكرد شناختى مرتبط است. همجنين نقص شناختى در توجه و عملكرد حافظه كلامى مشاهد

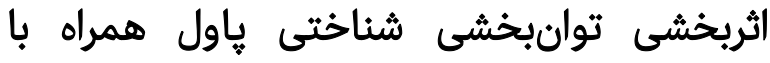
نوروفيدبك بر كاركردهاى اجرايى، حافظه و توجه

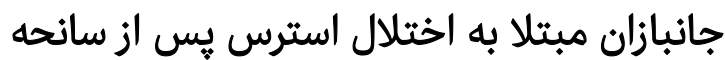

PhD * مهدى صحراگرد كروه روانشناسى، دانشكده علوم انسانى، واحد بهارستان، دانشگاه يِيام نور، تهران، ايران

PhD احمد علييور ايران كروه روان شناسى، دانشكده علوم انسانى، دانشكاه يِيام نور، تهران، ايران حسين زارع PhD كروه روانشناسى، دانشكده علوم انسانى، دانشكاه يِيام نور، تهران، ايران

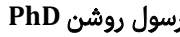
كروه روانشناسى، دانشكده علوم انسانى، دانشگاه شاهد، تهران، ايران

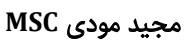
مركز تحقيقات مهندسى و علوم يزشكى جانبازان، تهران، ايران

جكيده اهداف: PTSD مجموعهاى از نشانههاى مستمر و مكرر است كه يس از راز تجربه

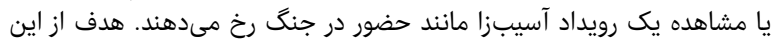

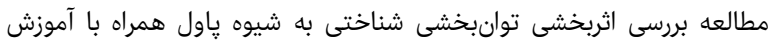

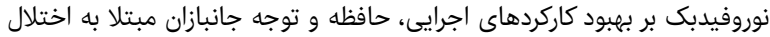

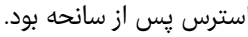

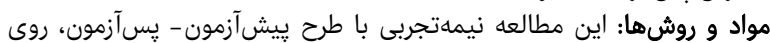

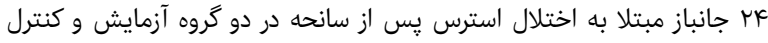

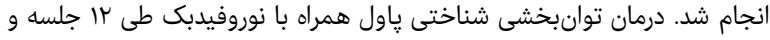

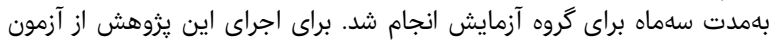

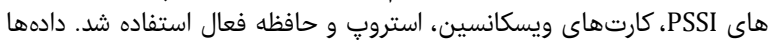

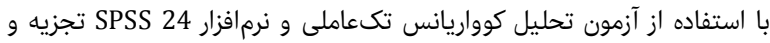

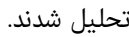

يافتهها: با كنترل اثرات ييشآنيآزمون، بين كروه آزمايش و كنترل از نظر نمره كلى

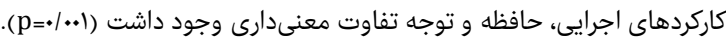

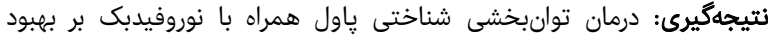

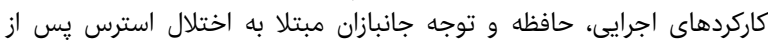

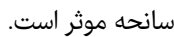
كليدواثهها: اختلالات استرس يس إز سانحه، توانبخشى، نوروفيدبك، كاركردهاى اجرايى،

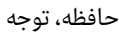

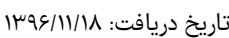

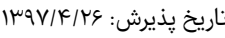

mahdisahragard@gmail.com :نويسنده مسئول:

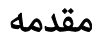

اختلال استرس يس از سانحه (PTSD) تا سال •191 جزء طبقه

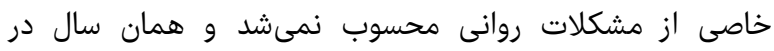

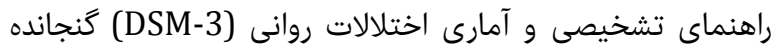

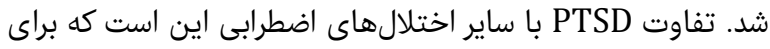

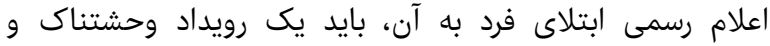

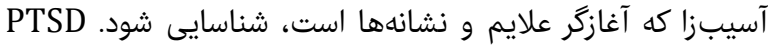

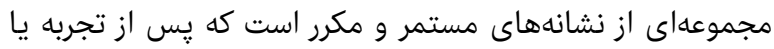

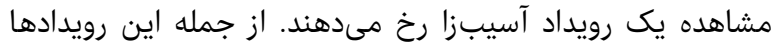

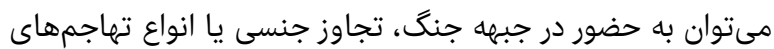

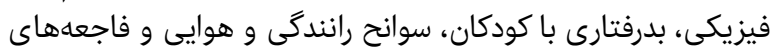

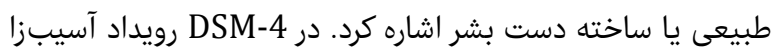

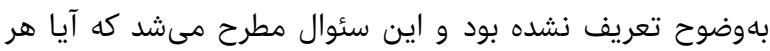

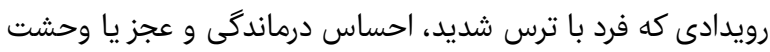

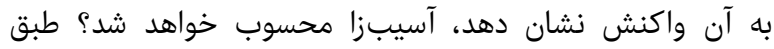




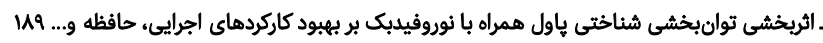

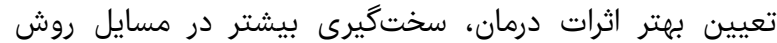

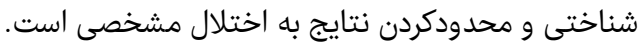

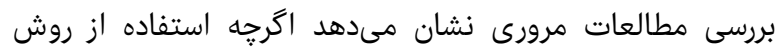

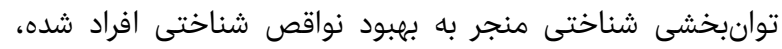

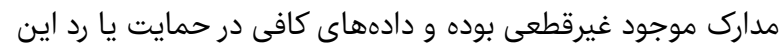

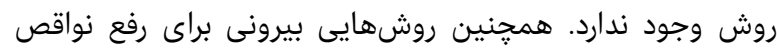

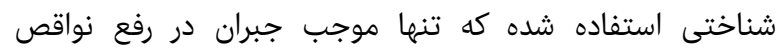

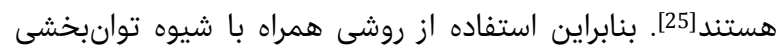

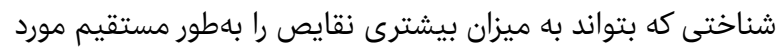

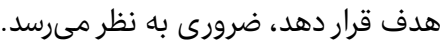
از جمله روشهايى كه در سالهایى به نظرئ اخير مورد توجه قرار گرفته،

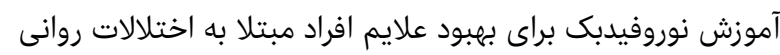

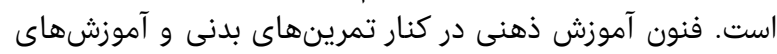

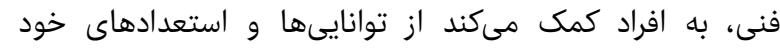

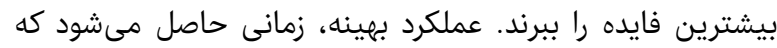

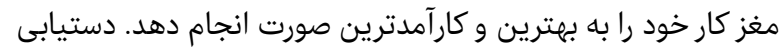

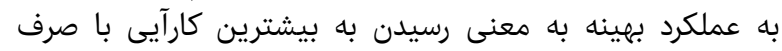

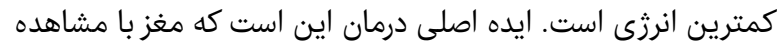

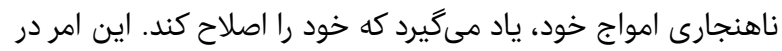

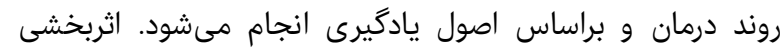

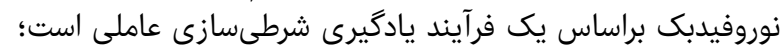

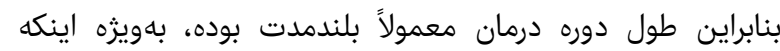

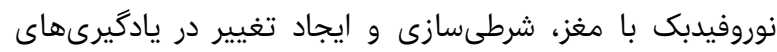

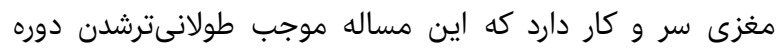

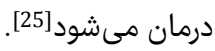

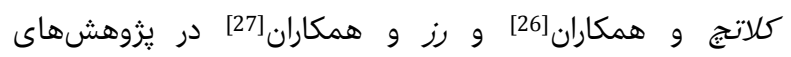
جداكانهاى با استفاده از امآرآى كاركردى (fMRI) تغييرات

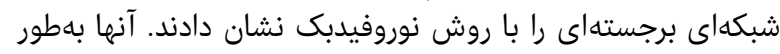

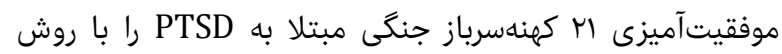

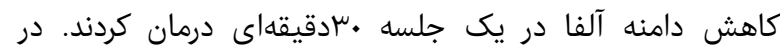

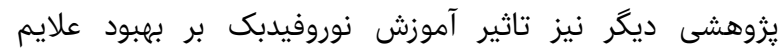

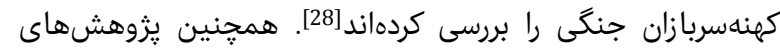

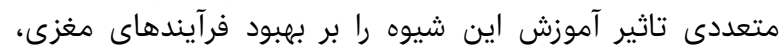

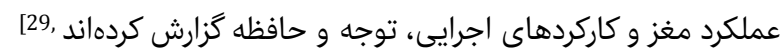

بهمنظور درمان جانبازان مبتلا به PTSD، لازم است شيوهانهاى

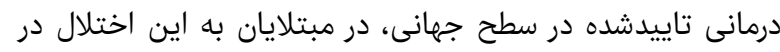

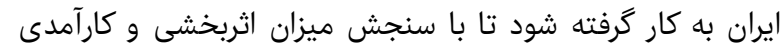

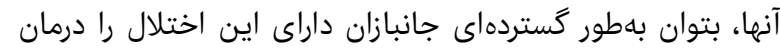

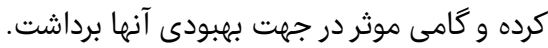

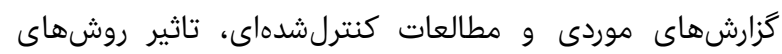

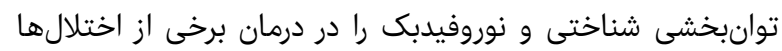

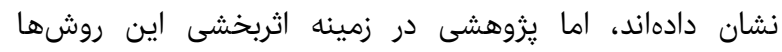

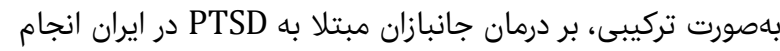

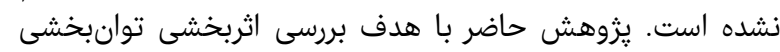

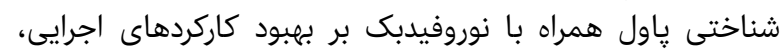

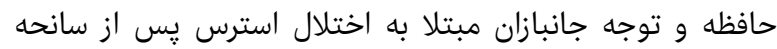

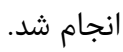

\section{مواد و روشها}

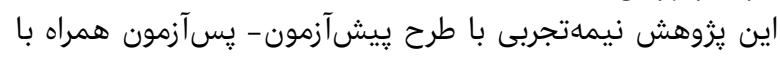

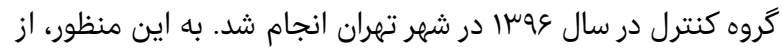

مىشود. در مطالعات اخير وجود نقص ويزهاى در فرآيندهاى

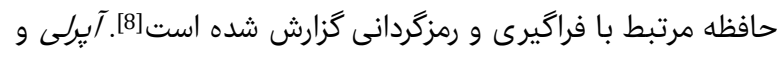

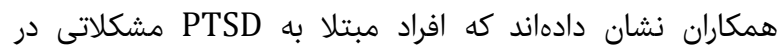

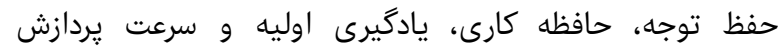

دارند [4].

نقايص شناختى همه بيماران را به درجات متفاوت تحت تاثير قرار

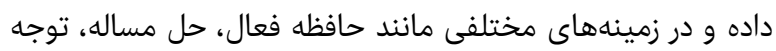

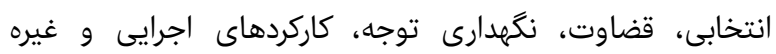

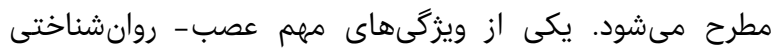

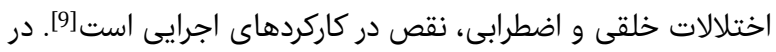

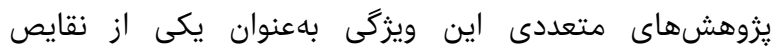

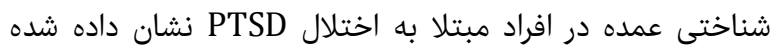

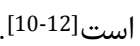

اگرجه PTSD اثر قابل توجهى بر شناخت عمومى ندارد، تحقيقات

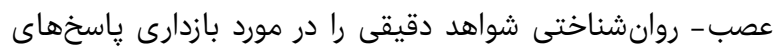

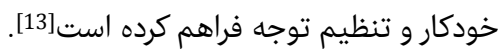

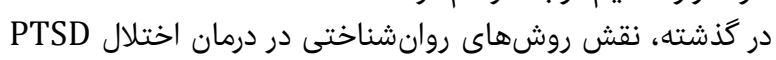

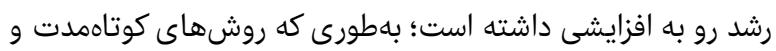

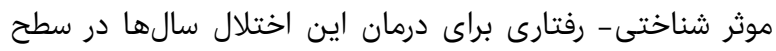

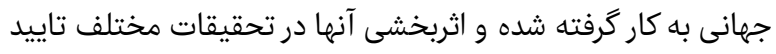

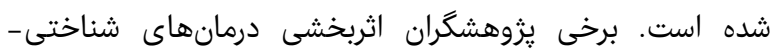

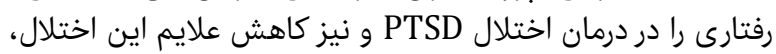

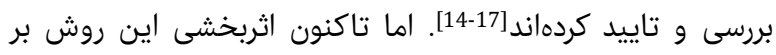

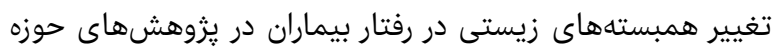

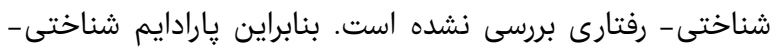

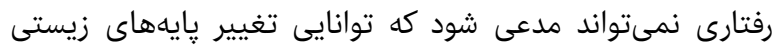
اختلالات روانى را دارد [18].

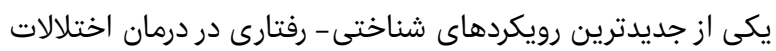

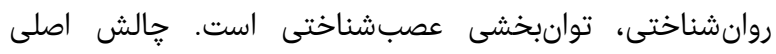

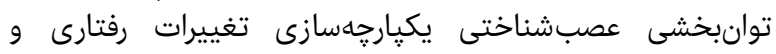

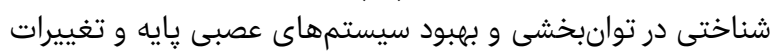

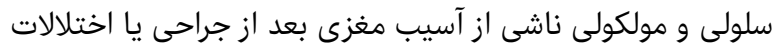

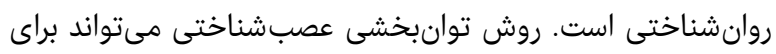

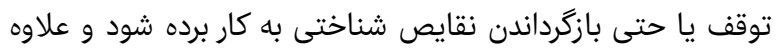

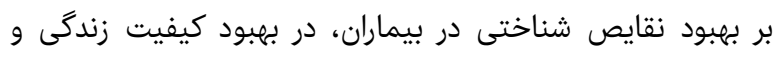

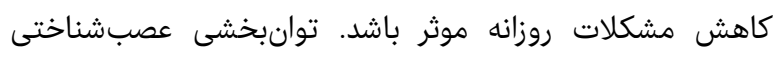

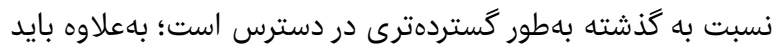

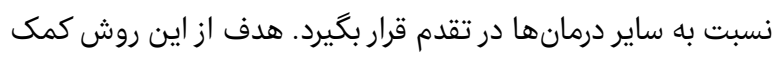

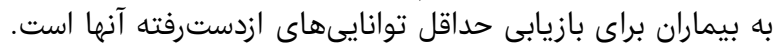

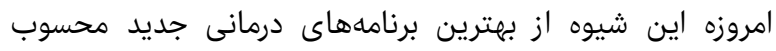

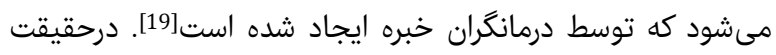

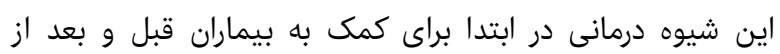

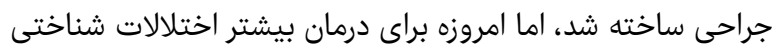

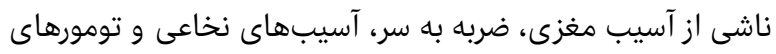

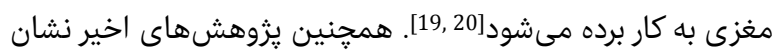

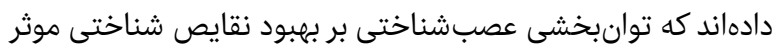

است[19, 21-23.

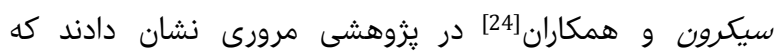

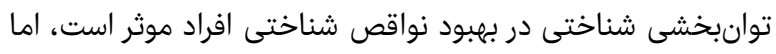

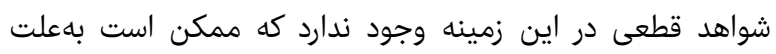

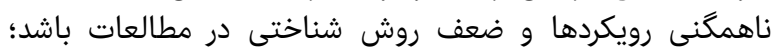

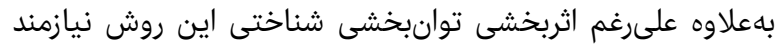


قرمز، آبى و زرد كذاشته شده است و از معاينهشونده خواسته

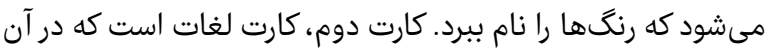

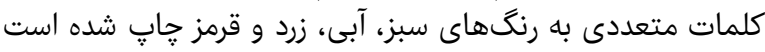

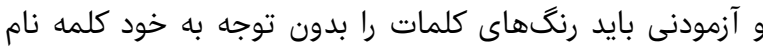

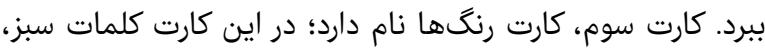

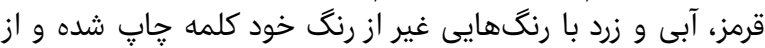

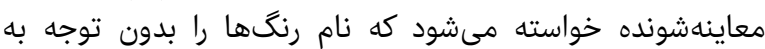

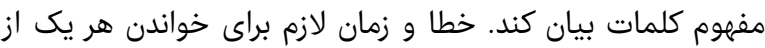

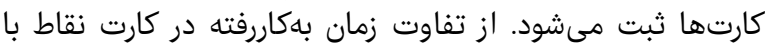

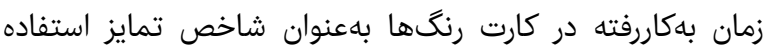

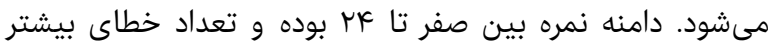

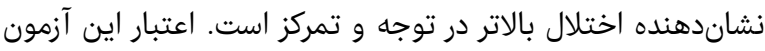

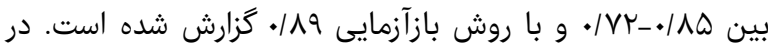

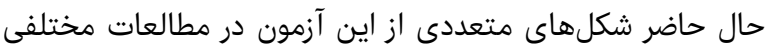

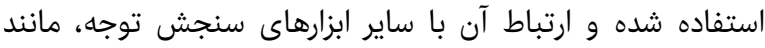

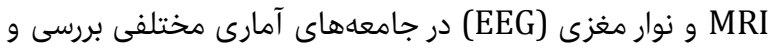

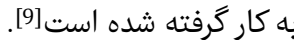

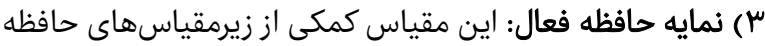

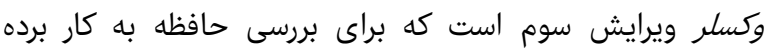

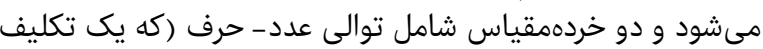

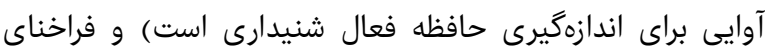

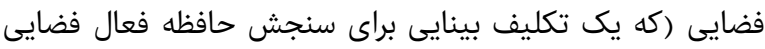

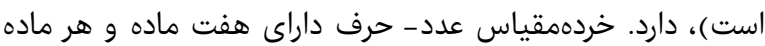

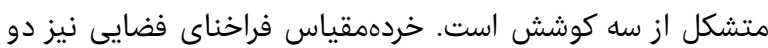

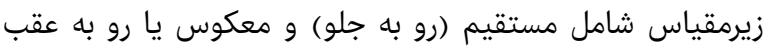

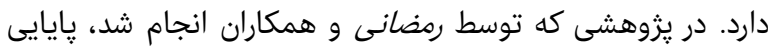

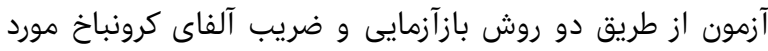

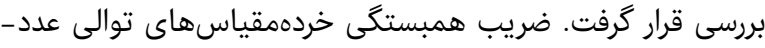

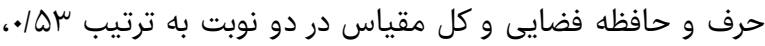

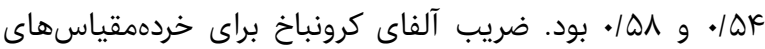

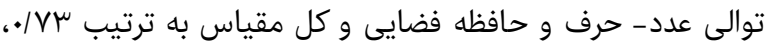

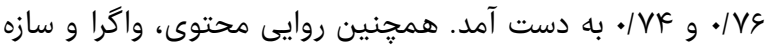
آزمون نيز تاييد شد [34].

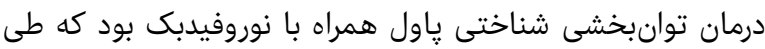

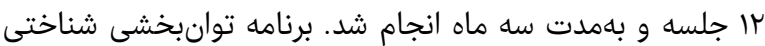

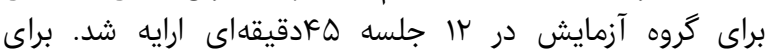

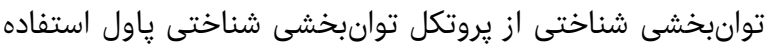

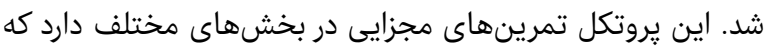

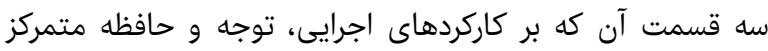

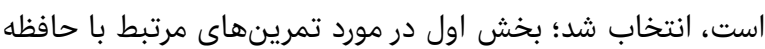

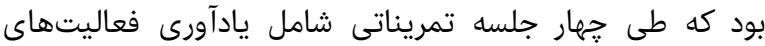

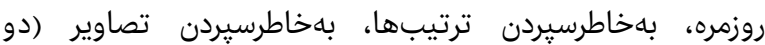

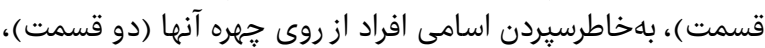

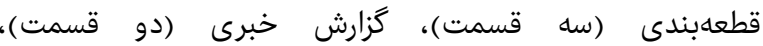
بهخاطرسيردن شمارهها و استفاده از وسايل كمكى تركى حافظه انجام

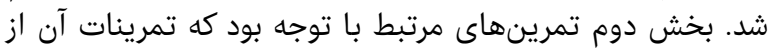

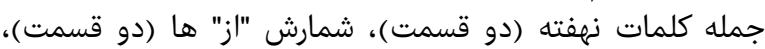

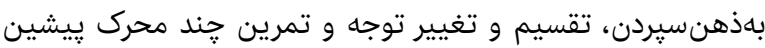

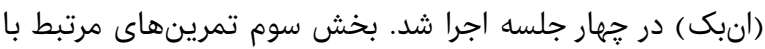

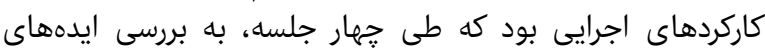

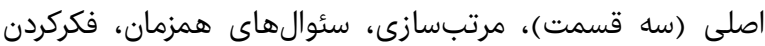

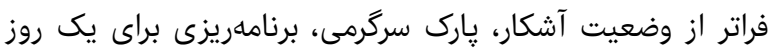

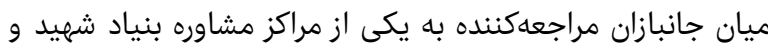

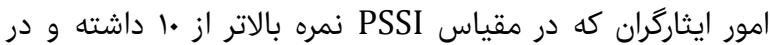

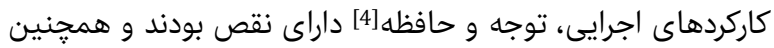

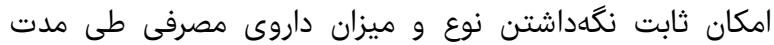

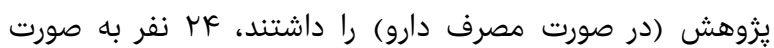

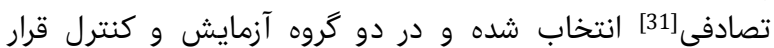

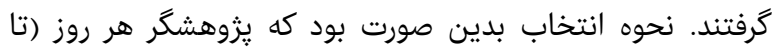

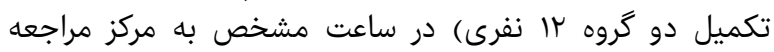

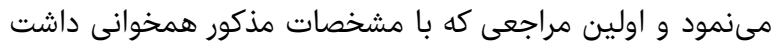

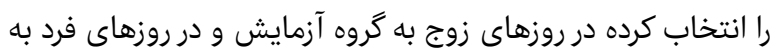

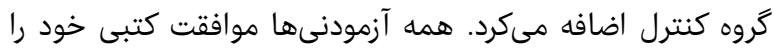

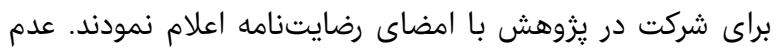

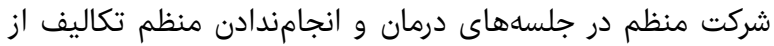

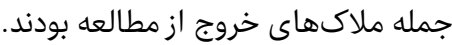

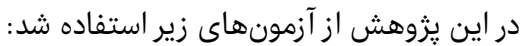

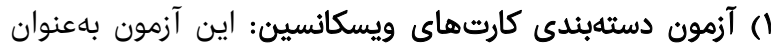

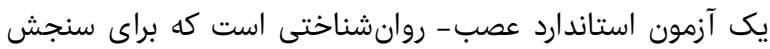

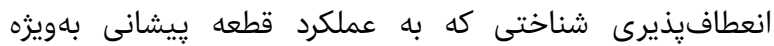

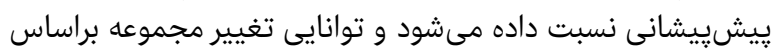

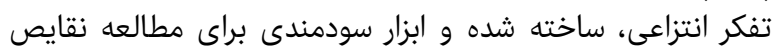

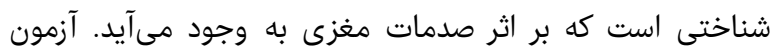

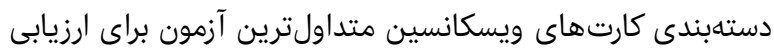

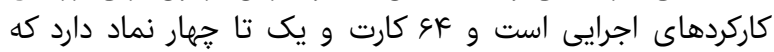

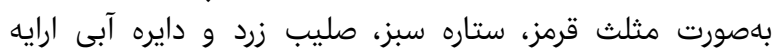

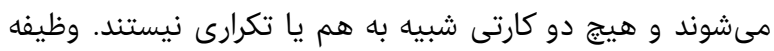

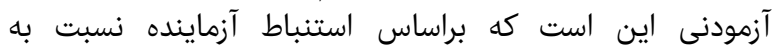

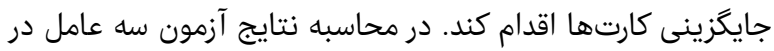

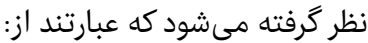

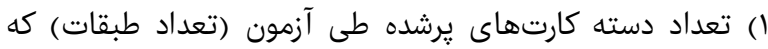

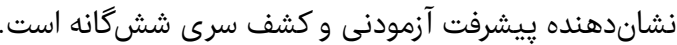

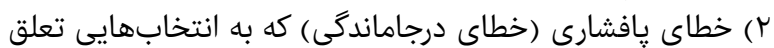

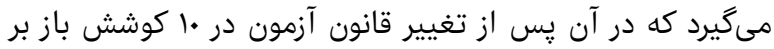

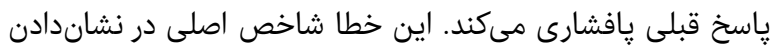

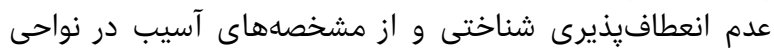
يشتى - جانبى قشر يرهفرونتال است است.

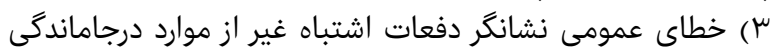
در انتخاب صحيح دسته كارتهاي استر دئ.

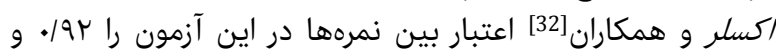

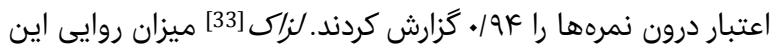

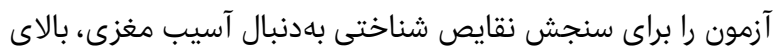

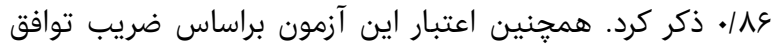

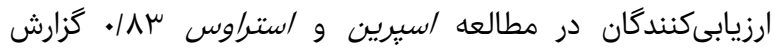

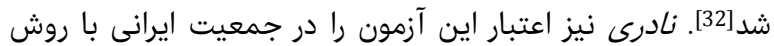

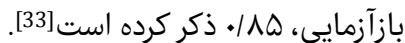

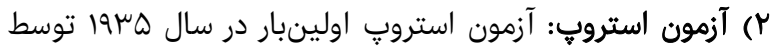

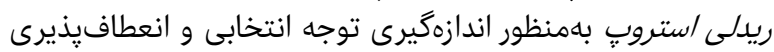

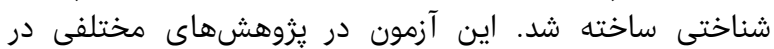

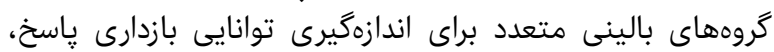

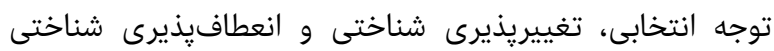

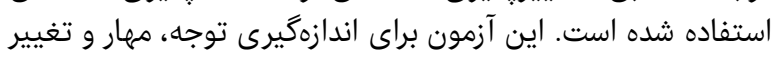

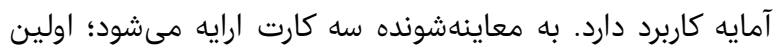

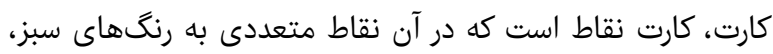




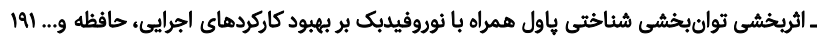

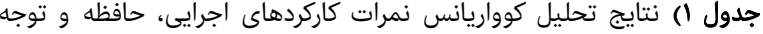

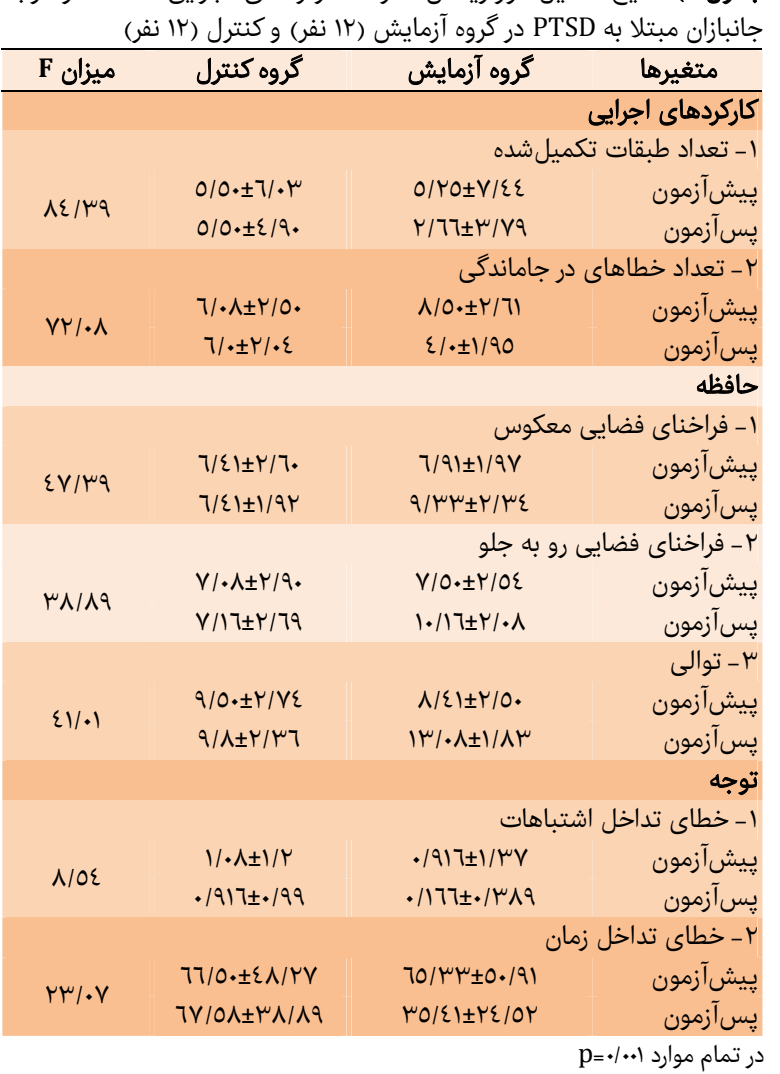

بحث

يزوهش حاضر با هدف بررسى تاثير درمان توانبخشى شناختى

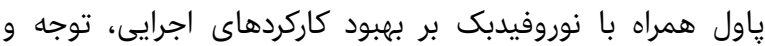

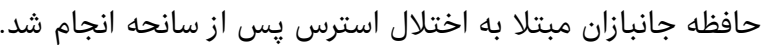

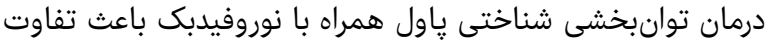

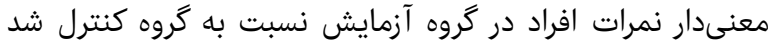

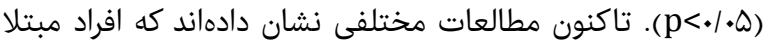

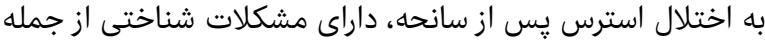

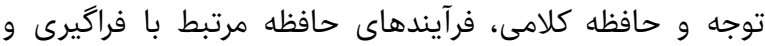

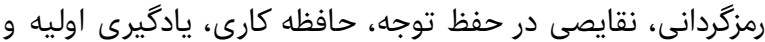

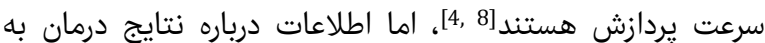
شيوه توانبخشى شناختى بسيار محدود است.

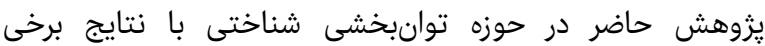

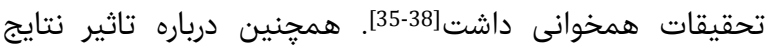

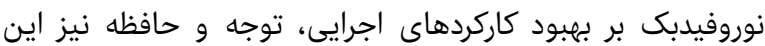

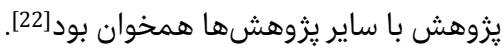

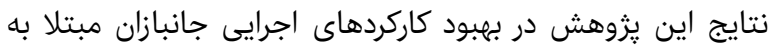

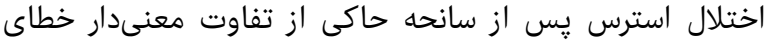

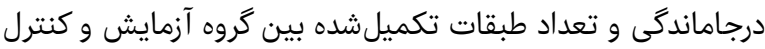

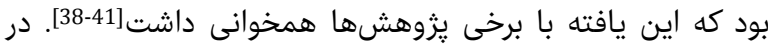

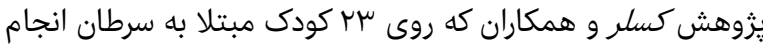
شد، برنامه توانبخشى شناختى بهطور معنىدارى موجب

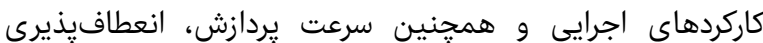

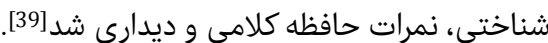

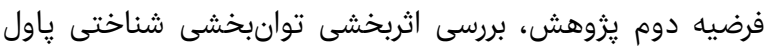

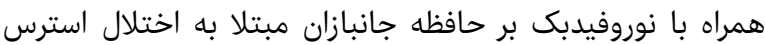

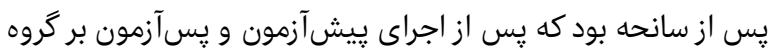

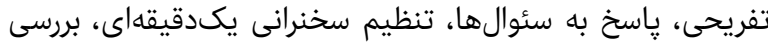

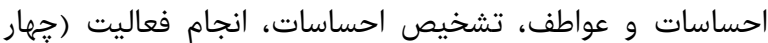

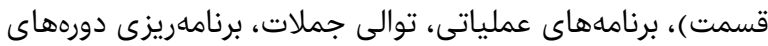

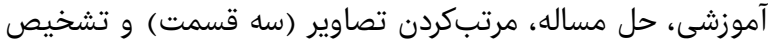

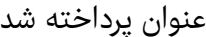
آموزش نوروفيدبك بعد از مداخله مداخله توانبخشى شناختى يكبار در

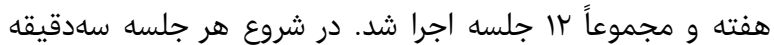

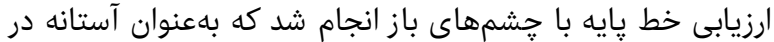

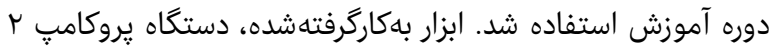

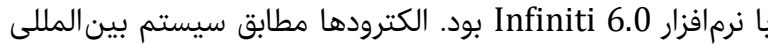

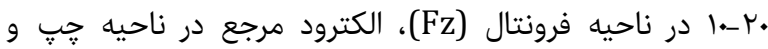

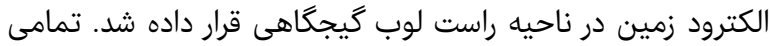

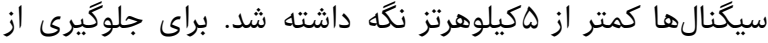

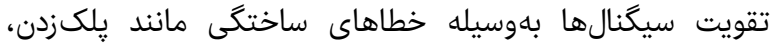

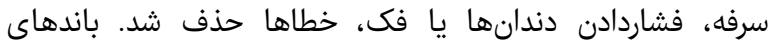

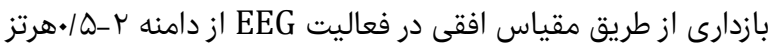

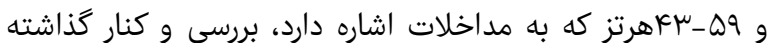

فيدبك شنيدارى - ديدارى به شكل فيلمهاى كوتاهى به كار گرفته

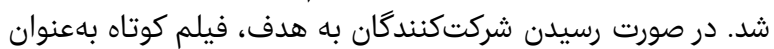

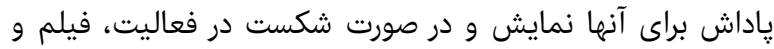

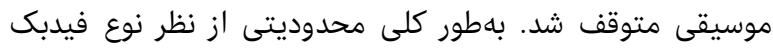

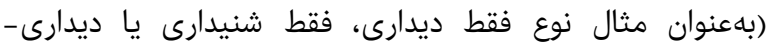

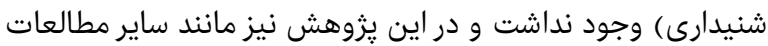

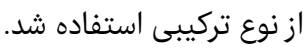

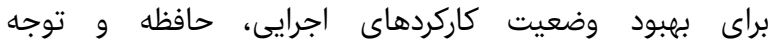

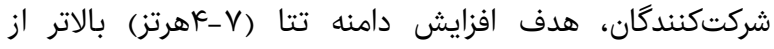

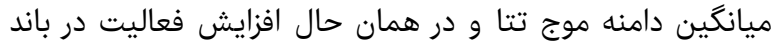

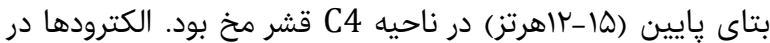

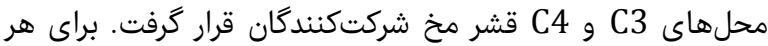

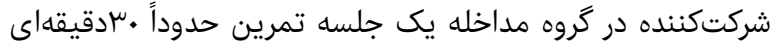

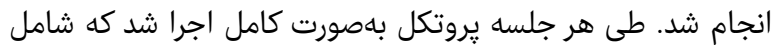

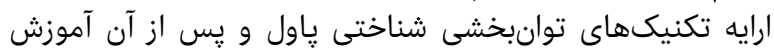

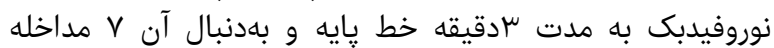

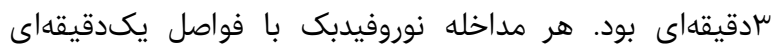

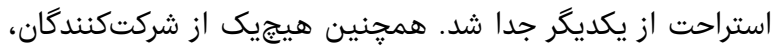

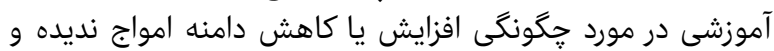

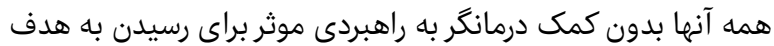

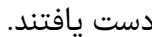

براى تجزيه و تحليل دادهها از نرمافزار SPSS 24 استفاده شد. از

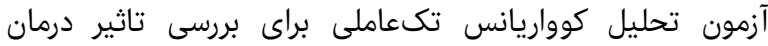

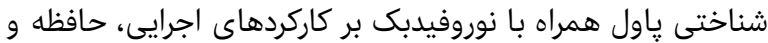
توجه استفاده شد.

\section{يافتهها}

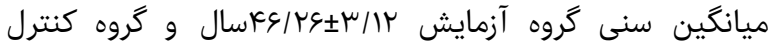

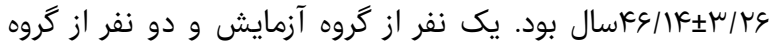

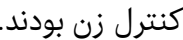

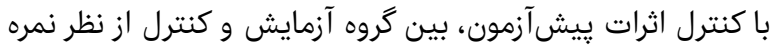

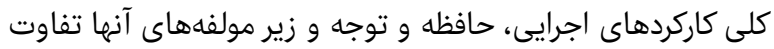

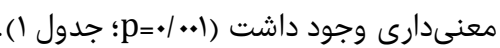


تيزوهشكران همكارى كردند، سياسگذارى مى شود.

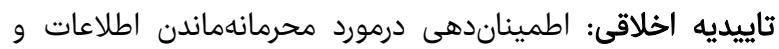

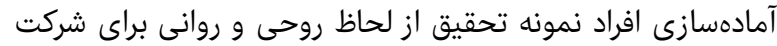

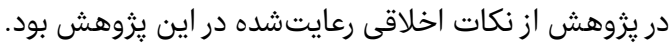

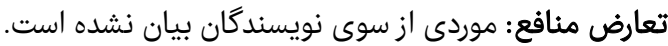

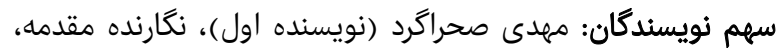

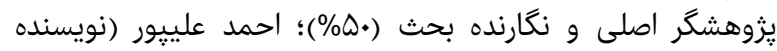

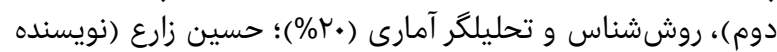

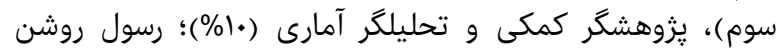

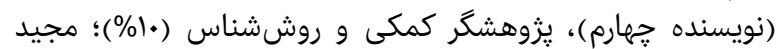

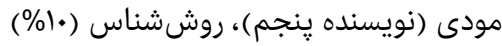

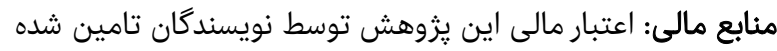

1- Ganji M. Mental disorders (DSM5). 1 ${ }^{\text {st }}$ Edition. Tehran: Savalan; 2014. p. 115-6. [Persian]

2- Donyavi V, Shafighi F, Rouhani SM, Hosseini S, Kazemi J, Arghanoun S, et al. The prevalence of PTSD in conscript and official staff of earth force in Tehran during 2005-6. Ann Mil Health Sci Res. 2007;5(1):1121-5. [Persian]

3- Tavallaie SA, Assari Sh, Najafi M , Habibi M, Ghanei M. Study of sleep quality in chemical-warfare-agents exposed veterans. J Mil Med. 2005;6(4):241-8. [Persian]

4- Aupperle RL, Melrose AJ, Stein MB, Paulus MP. Executive function and PTSD: Disengaging from trauma. Neuropharmacology. 2012;62(2):686-94.

5- Nardo D, Hogberg G, Jeffery C, Leong Looi JC, Larsson $\mathrm{S}$, Hallstrom $\mathrm{T}$, et al. Gray matter density in limbic and paralimbic cortices is associated with trauma load and EMDR outcome in PTSD patients. J Psychiatr Res. 2010;44(7):477-85.

6- Taylor SF, Liberzon I. Neural correlates of emotion regulation in psychopathology. Trends Cogn Sci. 2007;11(10):413-8.

7- Looi JC, Maller JJ, Pagani M, Hogberg G, Lindberg O, Liberg B, et al. Caudate volumes in public transportation workers exposed to trauma in the Stockholm train system. Psychiatry Res. 2009;171(2):138-43.

8- De Jongh A, Ernst R, Marques L, Hornsveld H. The impact of eye movement and tones on disturbing memories involving PTSD and other mental disorders. J Behav Ther Exp Psychiatry. 2013;44(4):477-83.

9- Molaie M, Hatami J, Rostami R. Evaluation and comparison of executive functions in patients with major depressive disorder and obsessive-compulsive disorder with healthy people. Adv Cogn Sci. 2014;16(3):61-71. [Persian]

10- Ziady LG. The association between complex trauma and executive functioning among an adolescent inpatient population [Dissertation]. Boston: Northeastern University; 2012. pp. 115-6.

11- Polak AR, Witteveen AB, Reitsma JB, Olff MA. The role of executive function in posttraumatic stress disorder: A systematic review. J Affect Disorder. 2012;141(1):11-21.

12- Olff MA, Polak AR, Witteveen AB, Denys D. Executive function in posttraumatic stress disorder (PTSD) and the influence of comorbid depression. Neurobiol Learn Mem. 2014;112:114-21.

13- Kershner AM, Kimble J. Genome-wide analysis of

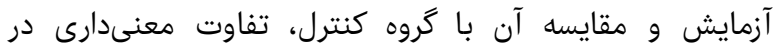

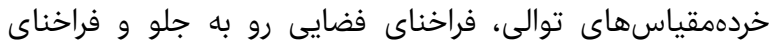

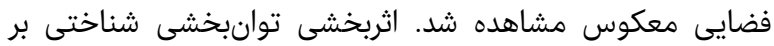

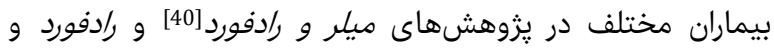

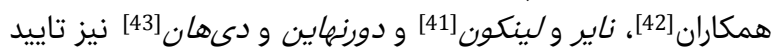

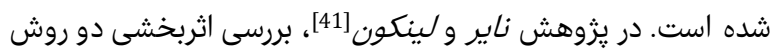

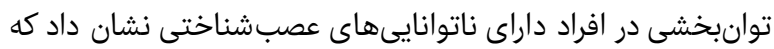

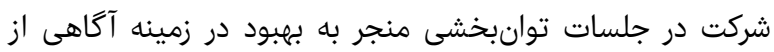

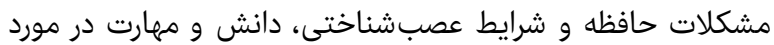

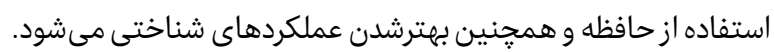

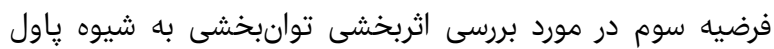

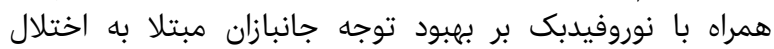

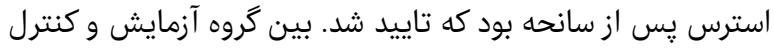

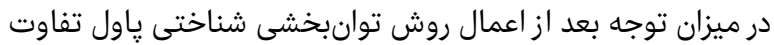

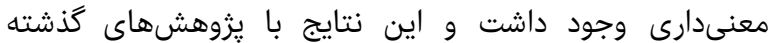

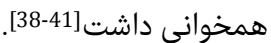

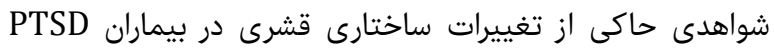

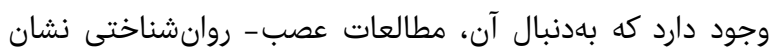

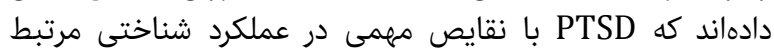

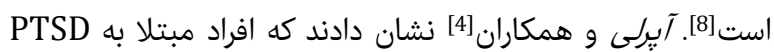

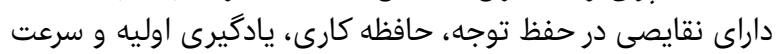

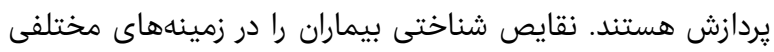

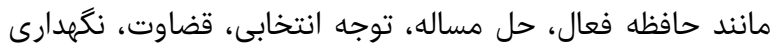

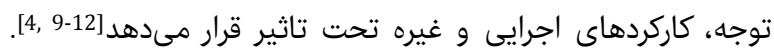

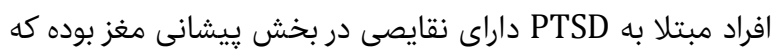

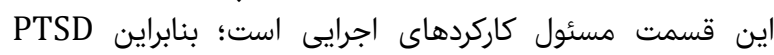

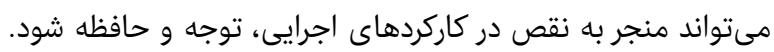

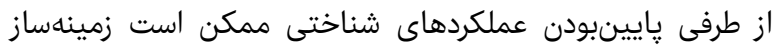

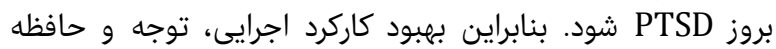

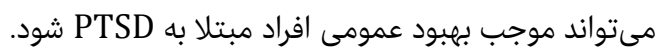

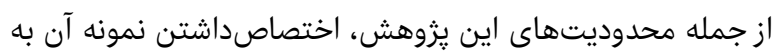

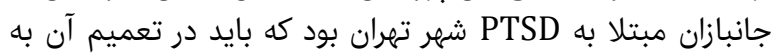

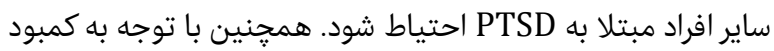

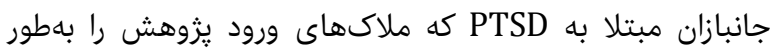

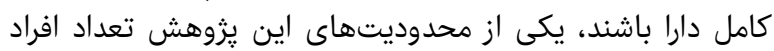

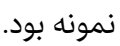
كسترش مطالعات در نمونههاى متنوع و هدايت مطالعات آينده در

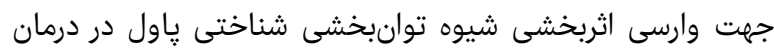

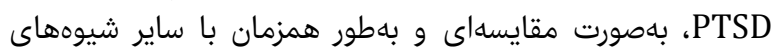

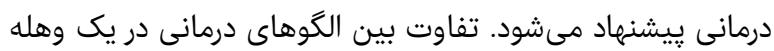

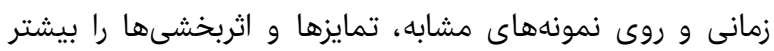

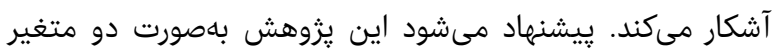

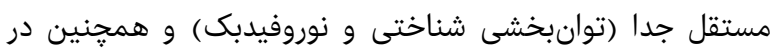

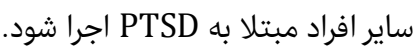

\section{نتيجه گيرى}

روش توانبخشى ياول همراه با نوروفيدبك بر بهبيود علايم

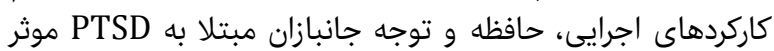

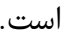

تشكر و قدردانى: صميمانه از همكارى تمامى جانبازان و كاركنان

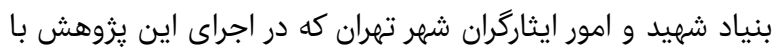


ـاثربخشى توانبخشى شناختى پاول همراه با نوروفيدبك بر بهبود كاركردهاى اجرايى، حافظه و... س19 emotion recognition and brain network connectivity in schizophrenia. Hum Brain Mapp. 2013;34(1):200-12.

28- Nelson DV, Esty ML. Neurotherapy of traumatic brain injury/posttraumatic stress symptoms in OEF/OIF veterans. J Neuropsychiatry Clin Neurosci. 2012;24(2):237-40.

29- Matsuda Y, Morimoto T, Furukawa S, Sato S, Hatsuse $\mathrm{N}$, Iwata $\mathrm{K}$, et al. Feasibility and effectiveness of a cognitive remediation programme with original computerised cognitive training and group intervention for schizophrenia: a multicentre randomised trial. Neuropsychol Rehabil. 2018;28(3):387-97.

30- Lee WK. Effectiveness of computerized cognitive rehabilitation training on symptomatological, neuropsychological and work function in patients with schizophrenia. Asia Pac Psychiatry. 2013;5(2):90-100.

31- Cozby PC. Methods in behavioral research. 10th Edition. New York: McGraw Hill; 2009. pp. 18-25.

32- Obeidi Zadegan A, Moradi A, Farnam R. Evaluation of cognitive functions in patients undergoing methadone treatment. Adv Cog Sci. 2009;10(3):75-81. [Persian] 33- Naderi N. Study of information processing and some neuropsychological functions of patients with obsessivecompulsive disorder [Dissertation]. Tehran: Anstitute Psychiatry of Tehran; 1998. pp. 98-9. [Persian]

34- Ramezani V, Moradi A, Ahmadi A. Active memory function in female students with high and normal depression symptoms. J Behav Sci. 2009;3(4):334-9. [Persian]

35- Matsuda K, Budisantoso T, Mitakidis N, Sugaya Y, Miura E, Kakegawa W, et al. Transsynaptic modulation of kainate receptor functions by $\mathrm{C} 1 \mathrm{q}$-like proteins. Neuron. 2016;90(4):752-67.

36- Miklos Z, Mychailyszyn M, Parente R. The efficacy of cognitive rehabilitation therapy: A meta-analytic review of traumatic brain injury and stroke cognitive language rehabilitation literature. Am J Psychiatry Neurosci. 2015;3(2):15-22.

37- Łojek E, Bolewska A. The effectiveness of computerassisted cognitive rehabilitation in brain-damaged patients. Polish Psychol Bull. 2013;44(1):31-9.

38- Lee J, Althuler L, Glahn DC, Miklowitz DJ, Ochsner K, Green MF. Social and nonsocial cognition in bipolar disorder and schizophrenia: Relative levels of impairment. Am J Psychiatry. 2013;170(3):334-41.

39- Kesler SR, Kent JS, O'Hara R. Prefrontal cortex and executive function impairments in primary breast cancer. Arch Neurol. 2011;68(11):1447-53.

40- Miller LA, Radford K. Testing the effectiveness of group-based memory rehabilitation in chronic stroke patients. Neuropsychol Rehabil. 2014;24(5):721-37.

41- Das Nair R, Lincoln NB. The effectiveness of memory rehabilitation following neurological disabilities: A qualitative inquiry of patient perspectives. Neuropsychol Rehabil. 2013;23(4):528-45.

42- Radford K, Lah S, Thayer Z, Say MJ, Miller LA. Improving memory in outpatients with neurological disorders using a group-based training program. J Int Neuropsychol Soc. 2012;18(4):738-48.

43- Doornhein K, De Haan EHF. Cognitive training for memory deficits in stroke patients. Neuropsychol Rehabil. 1998;8(4):393-400.
mRNA targets for Caenorhabditis elegans FBF, a conserved stem cell regulator. Proc Natl Acad Sci U S A. 2010;107(8):3936-41.

14- De Bont PA, Van Minnen A, De Jongh A. Treating PTSD in patients with psychosis: A within-group controlled feasibility study examining the efficacy and safety examining the efficacy and safety of evidencebased PE and EMDR protocols. Behav Ther. 2013;44(4):717-30.

15- Van Den Berg DP, Van Den Gaag M. Treating trauma in psychosis with EMDR: A pilot study. J Behav Ther Exp Psychiatry. 2012;43(1):664-71.

16- De Jongh A, Ernst R, Marques L, Hornsveld H. The impact of eye movement and tones on disturbing memories involving PTSD and other mental disorders. J Behav Ther Exp Psychiatry. 2013;44(4):477-83.

17- Leer A, Engelhard IM, Van Den Hout MA. How eye movements in EMDR work: Changes in memory vividness and emotionality. J Behav Ther Exp Psychiatry. 2014;45(3):396-401.

18- Jokić-Begić N. Cognitive-Behavioral Therapy and Neuroscience: Towards Closer Integration. 2010;19(2):235-54.

19- Zarghi A. Functional Neurosurgery and Neurocognitive Rehabilitation. Int Clin Neurosci J. 2014;1(2):43-7.

20- Zarghi A, Zali A, Ashrafi F, Moazaezi M. Emotional, behavioral and cognitive mechanisms in rehabilitation of romantic love neuro-cognitive process. Basic Res J Med Clin Sci. 2013;2(9):94-102.

21- Tomas P, Fuentes I, Roder V, Ruiz JC. Cognitive rehabilitation programs in schizophernia: Current status and perspectives. Int J Psicol Psicol Ter. 2010;10(2):191204.

22- Zaytseva Y, Korsakova N, Agius M, Gurovich I. Neurocognitive functioning in schizophernia and during the early phases of psychosis: Targeting cognitive remidation interventions. BioMed Res Int. 2013(2013);3:ID 819587.

23- D`Esposito M, Gazzaley A. Neurorehabilitation of executive function [Internet]. California: University of California, Berkeley; 2014. [cited 2016 Aug 5]. Available from:

https://pdfs.semanticscholar.org/5066/a4f91081a52fa3 b6ae1cf0bd916a34542bd6.pdf

24- Cicerone KD, Langenbahn DM, Braden C, Malec JF, Kalmar K, Fraas M, et al. Evidence-based cognitive rehabilitation: Updated review of the literature from 2003 through 2008. Arch Phys Med Rehabil. 2011;92(4):519-30.

25- Reis J, Schambra HM, Cohen LG, Buch ER, Fritsch B, Zarahn E, et al. Noninvasive cortical stimulation enhances motor skill acquisition over multiple days through an effect on consolidation. Proc Natl Acad Sci U S A. 2009;106(5):1590-5.

26- Kluetsch RC, Ros T, Théberge J, Frewen PA, Calhoun VD, Schmahl C, et al. Plastic modulation of PTSD restingstate networks and subjective wellbeing by EEG neurofeedback. Acta Psychiatr Scand. 2014;130(2):12336.

27- Ruiz S, Lee S, Soekadar SR, Caria A, Veit R, Kircher T, et al. Acquired self-control of insula cortex modulates 